University of Nebraska - Lincoln

DigitalCommons@University of Nebraska - Lincoln

2009

\title{
Mining Sky Surveys for Astrophysically Interesting Variable Stars: The Cepheid Period Range
}

\author{
Edward G. Schmidt \\ University of Nebraska at Lincoln, eschmidt1@unl.edu
}

Follow this and additional works at: https://digitalcommons.unl.edu/physicsschmidt

Part of the Physics Commons

Schmidt, Edward G., "Mining Sky Surveys for Astrophysically Interesting Variable Stars: The Cepheid Period Range" (2009). Edward Schmidt Publications. 42.

https://digitalcommons.unl.edu/physicsschmidt/42

This Article is brought to you for free and open access by the Research Papers in Physics and Astronomy at DigitalCommons@University of Nebraska - Lincoln. It has been accepted for inclusion in Edward Schmidt Publications by an authorized administrator of DigitalCommons@University of Nebraska - Lincoln. 


\title{
Mining Sky Surveys for Astrophysically Interesting Variable Stars: The Cepheid Period Range
}

\author{
Edward G. Schmidt \\ Department Physics and Astronomy, University of Nebraska-Lincoln, Lincoln, NE 68588, USA
}

\begin{abstract}
We have undertaken a project to discover new type II Cepheids in two large-area sky surveys. However, most of the candidates from the surveys differ from type II Cepheids in their amplitude and period distributions and they form a separate, cooler sequence relative to the Cepheids in a temperature - gravity plot. We refer to these objects as "Cepheid-like" stars. Many exhibit unstable pulsation which we illustrate with representative light curves.
\end{abstract}

Keywords: Cepheids, Population II Stars

PACS: 97.10.Sj; 97.20.Li; 97.20.Tr; 97.30.Gj

\section{INTRODUCTION}

The large-area photometric sky surveys that have appeared in recent years are an invaluable resource for variable star astronomy. The large numbers of variables, the superb temporal coverage and the accuracy they provide open opportunities to greatly increase the samples of variables of known types as well as to find new types of variables.

Since it can be argued that there should be many more type II Cepheids than are known (Wallerstein 2002), we undertook a search to identify new stars of this type. We selected a sample of nearly 500 stars from the variable star lists of Akerlof et al. (2000), based on data from a small portion of the Northern Sky Variability Survey, and of Pojmanski et al. (2005), based on the northern portion of the All Sky Automated Survey. Stars were selected on the basis of the periods $(1<\mathrm{P}<100$ days $)$ and classifications (periodic, pulsating stars) given in those lists together with the requirement that they be more that $10^{\circ}$ from the galactic plane (to ensure that any Cepheids found were type II Cepheids).

The NSVS survey lacks color information and the accuracy of the data from both surveys is inadequate for the reliable assessment of light curve shape and scatter. Because of this and because longer temporal coverage is helpful in determining periods and assessing the long-term behavior of the stars, we obtained new light curves in $\mathrm{V}$ and $\mathrm{R}$ at Behlen Observatory (Schmidt et al, 2007 and 2009, hereafter Papers I and II, respectively).
About $1 / 4$ of stars could be rejected as Cepheids on the basis light curve appearance (obvious detached binaries, constant stars, irregular variables, etc) or revised period leaving a sample of 370 candidates.

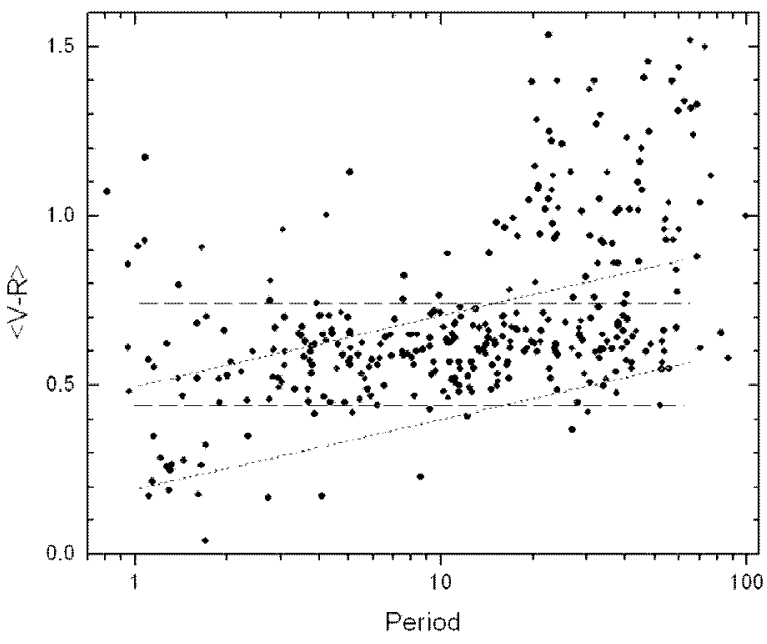

FIGURE 1. The mean colors of the candidate stars versus their periods. The slanted dotted lines mark the region of the known type II Cepheids and the horizontal dashed lines delineate the region of the Cepheid-like stars as discussed in the text.

To remove further non-Cepheids from the sample we plotted the mean $\langle\mathrm{V}-\mathrm{R}\rangle$ colors against period; see Figure 1. For comparison the slanted parallel lines outline the region in which most stars from a sample of known type II Cepheids lie (Schmidt et al, 2004, $2005 a, 2005 b$ ). It can be secn that while the points are 
scattered widely, a significant fraction, about $2 / 3$, occupy a relatively narrow, horizontal strip (delineated by the horizontal dashed lines) which partially overlaps the region of the type II Cepheids. We shall refer to these stars as "Cepheid-like" stars below on the assumption that they are not true type ПI Cepheids but are related to them.

In Figure 2 we plot the distributions of the mean amplitudes and periods for the Cepheid-like stars and for the known type II Cepheids. Although there is overlap in the amplitude distributions, it is clear that most of the Cepheid-like stars represent a distinct type of variable from the type II Cepheids. This conclusion is confirmed by the period distribution which lacks the gap between 3 and 10 days $(0.6<\log P<1.0)$ that is characteristic of type II Cepheids.
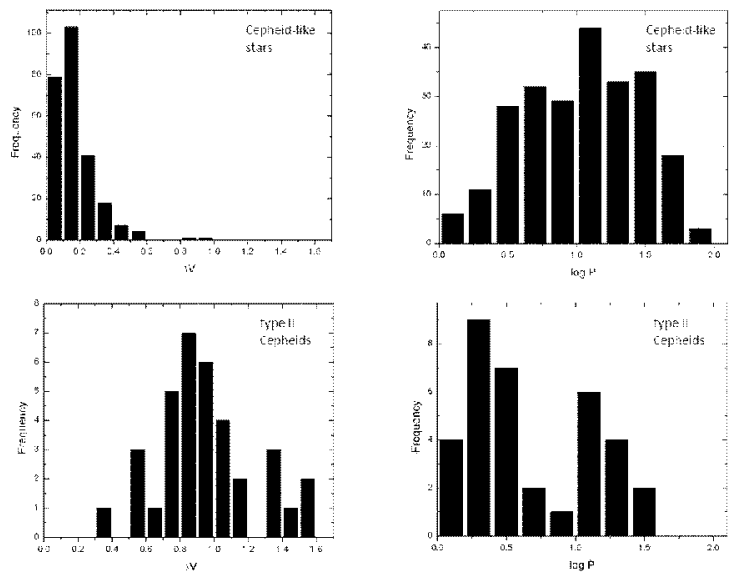

FIGURE 2. The frequency distributions of the amplitudes (left panels) and periods (right panels) for the Cepheid-like stars (top panels) and known type II Cepheids (lower panels).

\section{ATMOSPHERIC PARAMETERS}

We have obtained low resolution spectra with the GoldCAM spectrograph on the 2.1 meter telescope at Kitt Peak National Observatory for nearly all of the Cepheid-like stars as well as a sample of known Cepheids. These will be used to obtain temperatures, gravities and metallicities. The spectra for about $20 \%$ of the stars exhibited strong emission in $\mathrm{H} \alpha$. (as well as in $\mathrm{H} \beta$ or even $\mathrm{H} \gamma$ in a few cases). Such objects are likely to be active binaries and they were removed from the sample. While fewer than half of the spectra have been analyzed, preliminary results are shown in Figure 3. Gravity is a good proxy for luminosity, so the plot resembles the HR diagram.

We note that all of the known Cepheids lie in a hot sequence extending from $\log \mathrm{g} \sim 3$ and $\mathrm{T}_{\text {eff }} \sim 6000^{\circ}$ towards the upper right to $\log \mathrm{g} \sim 0.5$ and $\mathrm{T}_{\text {eff }} \sim 4500^{\circ}$. On the other hand, most of the Cepheid-like stars are in a parallel sequence approximately $700^{\circ}$ cooler.
While these results are very preliminary and there is no doubt contamination by other types of stars, binaries in particular, we conclude that the spectra confirm the above conjecture that the Cepheid-like stars are related to, but are distinct from, the type II Cepheids. Pulsational calculations are needed to identify the mechanism and mode of pulsation.

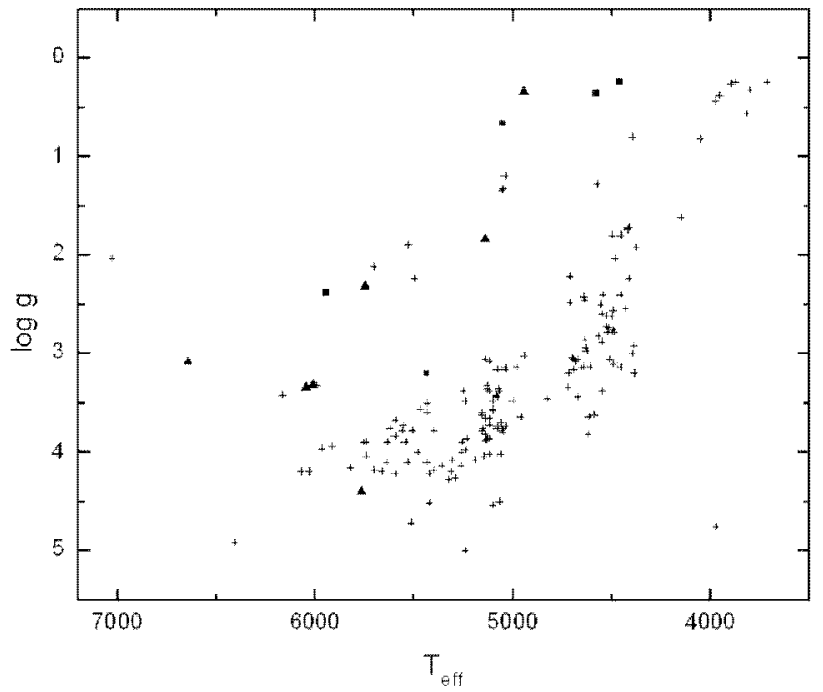

FIGURE 3. The atmospheric parameters derived from spectra. Plus signs denote Cepheid-like stars while squares and triangles represent type I and type II Cepheids, respectively.

\section{PULSATIONAL BEHAVIOR}

Variations in the periods, mean magnitudes, amplitudes, light curve shapes or a combination of these occur in about $70 \%$ of the Cepheid-like stars (see Papers $\mathrm{l}$ and II). This is consistent with the suggestion above that these stars may be post-AGB stars (Sasselov 1993). Sometimes these instabilities occur on short time scales, from cycle to cycle, while in other cases, the variation is relatively stable over a period of months.

Although it is usually difficult to discern patterns in this behavior, some interesting examples are illustrated below. The stars are all referred to by the running numbers employed in Paper II; Table 1 in that publication identifies them with the standard notation. For the plots below, the observations from the NSVS, ASAS and Behlen Observatory have been separated into groups that span at least an observing season. It can be seen that the light curves of these particular stars are relatively stable over a season or more and the changes in the pulsation occur on a time scale of a few years 


\section{Major Changes in Light Curve Shape}

During the Behlen observations, stars A133 and A201 exhibit symmetric, triangular minima with relatively flat maxima (see Figure 4). When plotted with twice the period, the minima are difficult to distinguish from eclipses. In fact, if it weren't for the fact that at the earlier epochs the light curves showed little evidence of eclipses, we would have been tempted to reject these stars from our sample. Instead, it seems likely that during the Behlen observations they were simply in a stage where they had light curves of the form designated as type A by Schmidt et al. (2004) and exemplified by AL Lyr and PP Aql.

These stars serve to warn us that there may be other type II Cepheids or Cepheid-like stars which have been misclassified as eclipsing stars in the sky surveys.
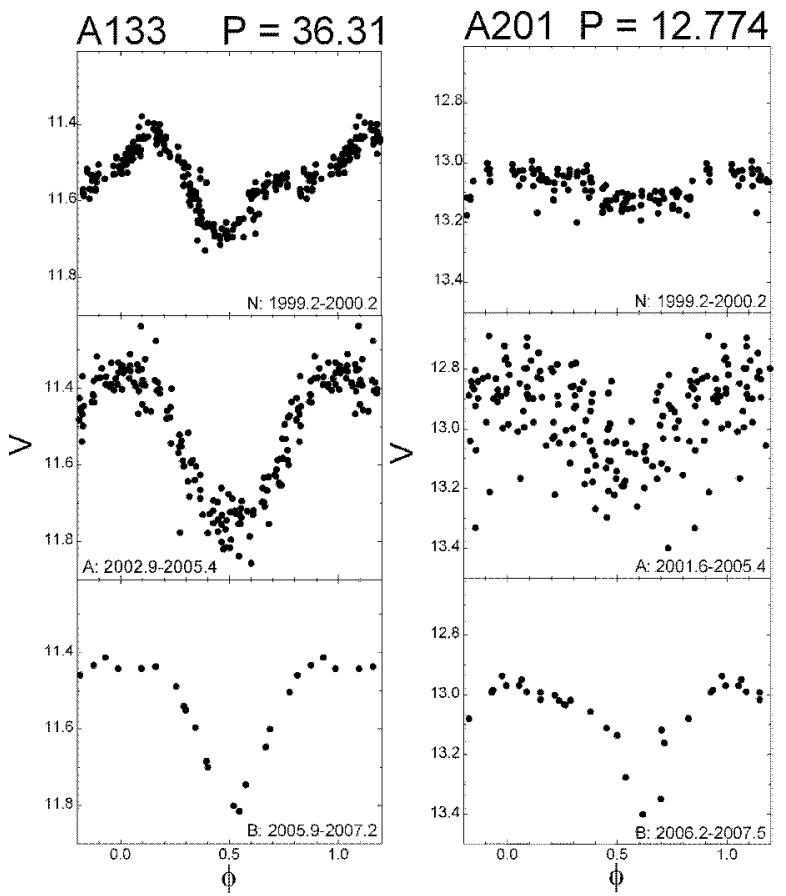

FIGURE 4. Phase diagrams for stars with changing light curve shapes. Each panel represents a contiguous subset of data. The subsets are identified by N, A or B to indicate data from the NSVS, the ASAS or Behlen Observatory, respectively. The letter is followed by the range of epochs, in years, included in the subset.

\section{Alternation of Cycles}

In Figure 5 we show three examples of stars with alternating cycles. In the NSVS data, A074 exhibits two nearly identical maxima and minima over a 4.7 day interval. However, one of the maxima is absent in the Behlen data and the ASAS data show obvious differences in the two minima. A262 exhibits two similar cycles in the second NSVS epoch and the ASAS data. While has no instances where one maximum is absent, there are clearly significant changes in the relative depths of the minima from one epoch to another, particularly in the Behlen data. A002 lacks an epoch where the two short cycles match but there are obvious changes among the maxima and minima of the various epochs.
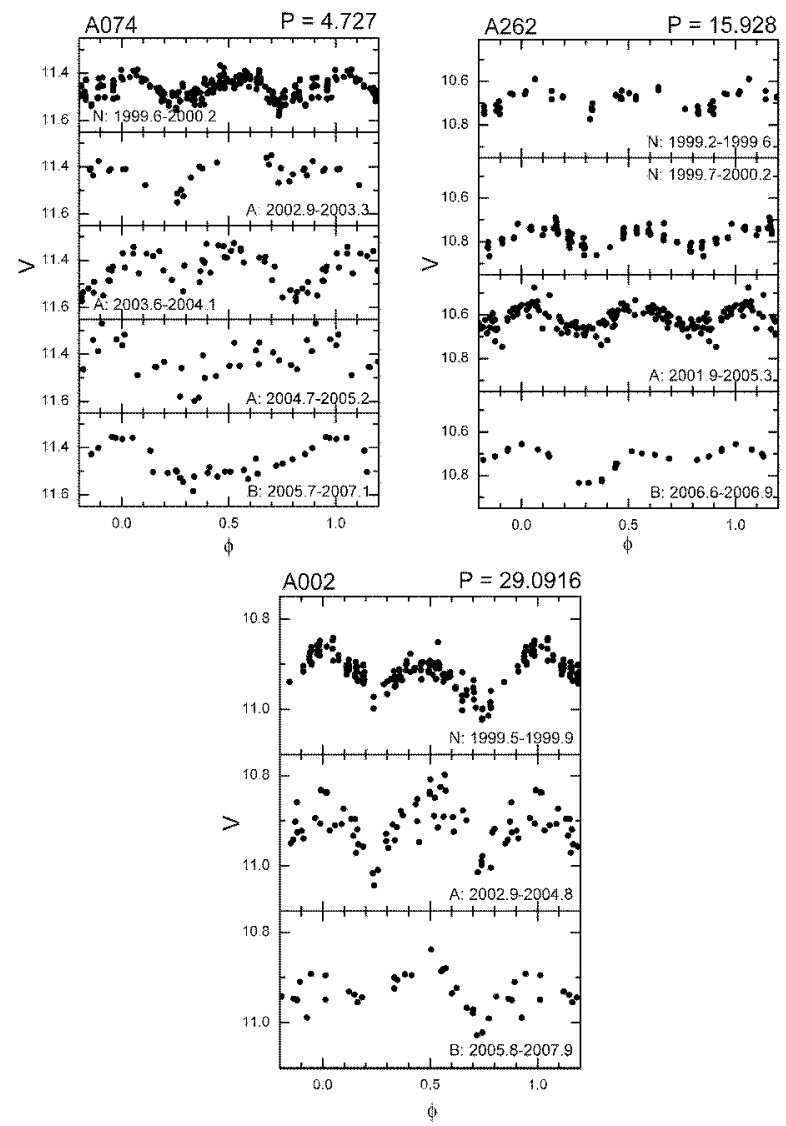

FIGURE 5. Phase diagrams for stars exhibiting cycle alternation. The data shown in each panel is identified in the same fashion as in Figure 4.

To some degree these light curves are reminiscent of the cycle alternation seen in the RV Tau stars. However, the amplitudes are too small and, except for A002, the periods too short for stars of this type. Additionally, the author is unaware of any instance similar to the apparent disappearance of one maximum in A074 among RV Tau stars.

\section{Cessation of Pulsation}

A number of stars may exhibit intervals without significant variability. A good example is A015, shown in Figure 6. It can be seen that the earlier data sets show variation with an amplitude of about 0.2 magnitudes, albeit with some changes in light curve 
shape. However, in the last set of data taken during the second season of observations at Behlen Observatory the star is constant with an rms scatter of 0.02 magnitudes; the pulsation has effectively stopped. While the explanation for this behavior is unclear, it may have the practical effect of causing some variables to be overlooked if the survey catches them during quiescence.

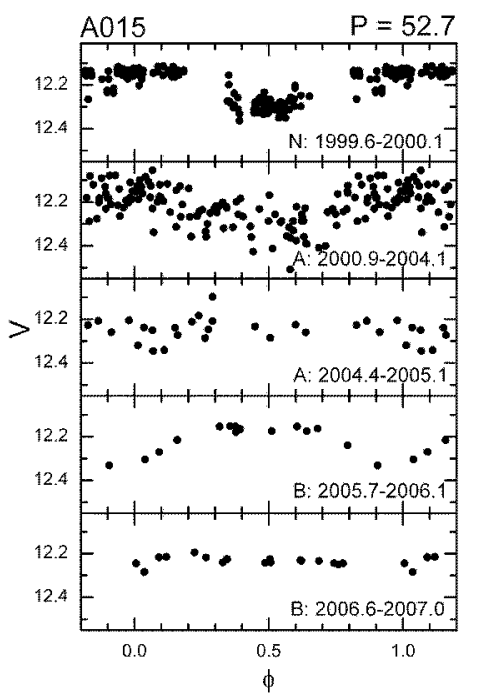

FIGURE 6. Phase diagrams for a star which stopped pulsating. The data in each panel is identified as in Figure 4.

\section{Future Prospects}

With the available photometry it is not possible to examine the detailed behavior of most of our stars. Even for the stars discussed above, our data is insufficient to guide the development of theoretical models for the behavior of these stars. However, it is quite possible that future surveys will provide sufficient temporal coverage to characterize the detailed behavior of these stars and lead to a theoretical understanding of that behavior.

\section{ACKNOWLEDGEMENTS}

The research described here made use of data from the All Sky Automated Survey and the Northern Sky Variability Survey. The author is grateful to the University of Nebraska for continued support for the operation of Behlen Observatory which was essential to this research.

\section{REFERENCES}

Akerlof, C. et al. 2000, Astron. J. 119, 1901

Pojmanski, G., Pilecki, B. and Szczygiel, D. 2005, Acta Astron. 55, 275
Sasselov. D.D. 1993, "Evolution from the AGB: Variability" in, Planetary Nebulae, edited by R. Weinberger and A. Acker, IAU Symposium 155, Kluwer Academic Publishers, Dordrecht, p. 259

Schmidt, E.G., Johnston, D., Langan, S. and Lee, K.M. 2004, Astron. J. 128, 1748

Schmidt, E.G., Johnston, D., Langan, S. and Lee, K.M. 2005a, Astron. J. 129, 2007

Schmidt, E.G., Johnston, D., Langan, S. and Lee, K.M. 2004, Astron. J. 130, 832

Schmidt, E.G., Langan, S., Rogalla, S. and Thacker-Lynn, L. 2007, Astron. J. 133, 665 (Paper I)

Schmidt, E.G., Hemen, B., Rogalla, D. and Thacker-Lynn, L. 2009, Astron. J. 137, 4598 (Paper II)

Wallerstein, G. 2002, PASP 114, 689 